Int. J. Speleol. 16 (1987), pp. 101-109

\title{
LES MÉSAVENTURES DES SOURCES DE L'ESTAVELLE ET DE L'INVERSAC EN LANGUEDOC MÉDITERRANÉEN
}

\author{
Bernard Gèze *
}

\begin{abstract}
RÉSUMÉ
L'Estavelle et l'Inversac sont deux sources célèbres du Languedoc méditerranéen (Sud de la France). La première a malheureusement servi de type pour les cavités karstiques alternativement absorban't ou dégorgeant des eaux, suivant les saison, ce qui n'a jamais été son cas. La seconde peut, au contraire, servir de modèle pour ce fonctionnement alternatif de perte ou d'émergence.
\end{abstract}

\section{SUMMARY}

The Estavelle and the Inversac are two celebrated springs in the mediterranean Languedoc (South of France). Unfortunately, the first one has been chosen as a type for the karstic cavities alternatively absorbing or discharging the waters, in accordance with the season, that had never been the case. On the opposit, the second one can be taken as model for this alternation as swallow hole or emergence.

Les sources de l'Estavelle et de l'Inversac sont citées depuis fort longtemps dans les ouvrages d" "Histoire Naturelle" des provinces méridionales de la France, car leur fonctionnement hydrologique a toujours beaucoup intrigué les observateurs. Elles se trouvent toutes deux dans le département de l'Hérault, à l'Ouest de Montpellier, la première dans la commune de Cabrières, près de l'extrémité orientale du massif ancien dit de la Montagne Noire, la seconde dans la commune de Balaruc-les-Bains, au bord de l'étang de Thau (fig. 1).

Leur description sommaire fera comprendre comment leurs étranges comportements ont provoqué des explications plutôt douteuses et comment l'accumulation de textes mal lus a entraîné d'invraisemblables er-

\footnotetext{
* Professeur honoraire de Géologie à l'Institut National Agronomique, Ancien Chargé de Cours d'Hydrogéologie à la Sorbonne, 11 rue Vauquelin, 75005 PARIS (France).
} 


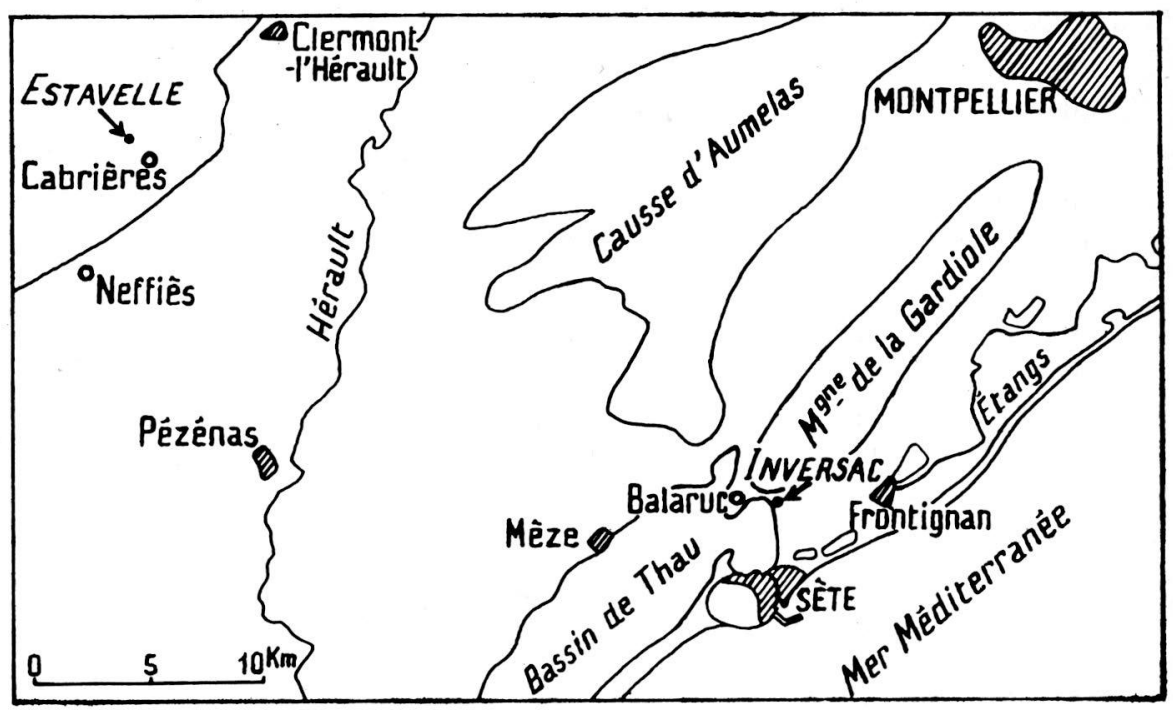

Fig. 1 - Situation géographique des sources de l'Estavelle et de l'Inversac.

Les Monts de Cabrières, le Causse d'Aumelas et la Montagne de la Gardiole sont à dominante karstique.

reurs, fâcheusement répétées par trop d'hydrologues et de karstologues. Mais leurs mésaventures sont aujourd'hui bien terminées, puisqu'aucune ne fonctionne plus!

\section{LA SOURCE DE L'ESTAVELLE}

Il semble que ce soit J. Fournet, alors professeur de Géologie à la Faculté des Sciences de Lyon, qui ait le premier, en 1859, attiré l'attention sur l'Estavelle dans son excellent travail sur l'Hydrographie souterraine, particulièrement consacré aux régions "caverneuses" du Jura, mais aussi du Bas-Languedoc où il avait étudié avec M. Graff, à partir de 1844, le petit bassin houiller de Neffiès, ainsi que les terrains paléozoiques qui le dominent dans ce que nous appelons maintenant les Monts de Cabrières, partie de la Montagne Noire célèbre par ses faunes fossiles allant de l'Ordovicien au Carboniferre et par sa tectonique d'une extrême complication.

C'est $1500 \mathrm{~m}$ au Nord-Ouest du village de Cabrières, par conséquent en plein Languedoc méditerranéen et non dans le Jura, que se trouve la 
source de l'Estavelle. Suivant les auteurs, son nom (qui s'écrit aussi Estabel) signifierait qu'il y avait une étable auprès d'elle, ou bien viendrait de l'Occitan "estervel", qui veut dire tourbillon. Quoi qu'il en soit, la source débouche sur un énorme dépôt de tuf calcaire $(1000 \mathrm{~m} \times 500 \mathrm{~m})$ formant un plateau en faible pente au-dessus de la vallée de la Boyne, dont le lit, déjà creusé, a été dévié par lui. D'après l'altitude relative du plateau (40 à $50 \mathrm{~m}$ ) et le fait que de petites cavités dans le tuf ont été habitées par l'ours des cavernes, on peut supposer que le début de la construction de cet édifice remonte à peu près au Quaternaire moyen et que la source a toujours débité une eau très riche en calcaire.

Or, le point de sortie se localise dans des schistes dinantiens (Viséen), sur le trajet d'une faille injectée de quartz, et non dans les calcaires et dolomies du Dévonien qui encadrent la région, ce qui est déjà curieux. On est conduit à penser que l'eau a suivi un assez long parcours en profondeur et n'a pu gagner l'extérieur qu'à la faveur de la faille, qui a servi soit de barrière, soit de drain.

Mais le plus étrange est que, malgré l'activité déployée forcément par la source pour construire le plateau de tuf, elle ne débite depuis longtemps que tout-à-fait rarement. Déjà Fournet notait quelle avait "vomi beaucoup d'eau' en 1856, mais qu'elle n'avait point fonctionné depuis dix ans. Personnellement, j'ai entendu parler de périodes de 25 ans sans qu'elle ait craché. La notice de la carte géologique de la France à 1/50.000

SW

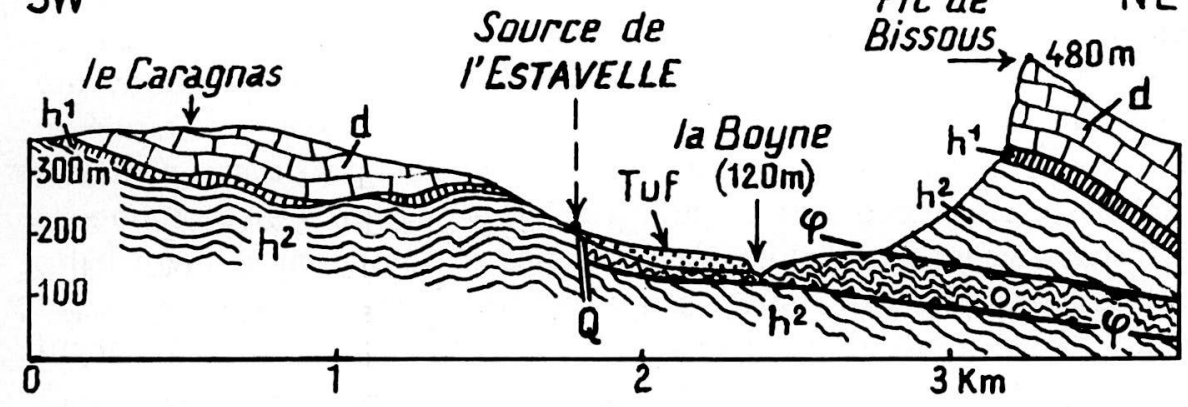

Fig. 2 - Coupe des chevauchements et séries renversées au voisinage de la source de l'Estavelle (l'échelle des hauteurs est double de celle des longueurs).

$\boldsymbol{\sigma}=$ schistes ordoviciens; $\mathrm{d}=$ calcaires et dolomies du Dévonien; $\mathrm{h}^{1}=$ lydiennes du Tournaisien; $h^{2}=$ schistes du Viséen (faciès culm); $Q=$ faille quartzifiée; $\varphi=$ surface de chevauchement.

Variantes orthographiques sur les cartes récentes: le Caragnas = les Cayraignasses; Estavelle

$=$ Estabel; Bissous $=$ Vissou. 
(Feuille Lodève, datant de 1982) précise: “l'Estabel est une source temporaire ne coulant que très exceptionnellement; son débit peut alors atteindre, en quelques jours, $1 \mathrm{~m}^{3} / \mathrm{s}$ et il est aussitôt suivi d'une période de tarissement pouvant s'étendre sur plusieurs mois".

Sans être troublé par un comportement aussi étrange, Fournet pense que l'on est en présence de "fontaines caractérisées par deux bouches en quelque sorte jumelles ... Dans tous les cas, la destination des unes, qui sont habituellement à sec, est de servir à l'évacuation du trop-plein des cavernes, du moment où l'orifice, dont l'écoulement est permanent, devient insuffisant par suite de l'exubérance des eaux ... Dans le Languedoc, ces bouches supplémentaires son désignées sous le nom d'Estavelles, dénomination que j'ai jugé à propos de généraliser, en l'appliquant à tous les pertuis du même ordre, disséminés dans le autres contrées'.

Quelques pages plus loin dans son mémoire, Fournet étudie des sources proches de Porrentruy, dans les calcaires du Jura franco-suisse et dit qu'en remontant une vallée "aux sources pérennes succède une première estavelle, puis viennent des estavelles d'estavelles, largement espacées, de plus en plus intermittentes, conformément à leurs hauteurs, et il me semble qu'un pareil enchaînement est suffisamment démonstratif pour ne plus rien laisser à désirer à l'égard de la parfaite solidarité de ces divers débouchés'.

Il est donc parfaitement clair que, pour Fournet, créateur du terme, une estavelle est une source de trop-plein fonctionnant temporairement au-dessus d'une source pérenne.

Mais a-t-il eu raison de prendre comme modèle d'un tel fonctionnement l'Estavelle type de Cabrières? Ce n'est pas évident pour plusieurs raisons: Au-dessous de sa bouche, il n'existe que de fort petites sources qui ne paraissent pas avoir le moindre rapport avec elle. Ensuite, la rareté du fonctionnement paraît incompatible avec un rôle normal de trop-plein, car les précipitations ne sont tout de même pas négligeables sur les monts de Cabrières où les fortes pluies de printemps et d'automne relèvent habituellement le débit de toutes les sources. Des décades sans débordement d'un trop-plein paraissent invraisemblables, aussi bien qu'un dépôt de tuf sans un écoulement assez régulier.

Depuis une douzaine d'années, on est certain qu'il n'y a plus eu de débordement, mais la raison en est évidente: un forage, profond de $55 \mathrm{~m}$ a été exécuté au voisinage du griffon et, dans les fissures des schistes sous la carapace de tuf, a rencontré de l'eau en quantité suffisante pour assurer l'alimentation du village de Cabrières. Cette eau a tous les caractères 
d'une eau karstique, ce qui confirme l'hypothèse d'une provenance relativement profonde, mais sans que l'on puisse garantir l'origine dans l'un ou l'autre des paquets de dolomies dévoniennes situés dans des position tectoniques particulièrement compliquées aux alentours. De toutes façons, l'exemple de l'Estavelle a été vraiment mal choisi par Fournet pour désigner de simples trop-pleins.

La fâcheuse histoire du terme "estavelle" ne s'arrête malheureusement pas là. Dans son célèbre ouvrage "Les Abîmes", E.A. Martel cite un peu en vrac des exemples de trop-pleins par lesquels "l'eau interne s'extravase" et a la mauvaise idée de mentionner les "Estavelles de Porrentruy" (puisées dans Fournet) en même temps que le "lac de Zirknitz en Carniole", dont il précise bien, quelques chapitres plus loin, le fonctionnement alternatif, rempli ou vidé par les cavités y aboutissant.

C'est alors que Jovan Cvijic, après avoir lu un peu vite les textes antérieurs, écrit: "dans les profondes dépressions karstiques il y a des fissures et des avens qui fonctionnent alternativement comme sources ou comme gouffres absorbants ou ponors; j'ai proposé de les appeler "estavelles" en utilisant la dénomination que Fournet a donné à une source semblable".

Cette désastreuse initiative explique pourquoi le terme est largement employé dans la littérature géographique de langues slaves, d'où elle nous est revenue au point que Marjorie Sweeting écrit: "The term estavelle was first used in the Jura but is now used fairly widely for a hole which is at one time of the year a swallow hole and an another time a spring" et que le lexique de J. Margat, publié pourtant en France, donne pour estavelle la définition: "orifice, en terrain karstique, alternativement absorbant ou émissif, selon les saisons".

En définitive, on se trouve en présence d'un terme mal choisi par Fournet pour signifier trop-plein temporaire, reproduit avec une errente géographique par Martel et mal compris au sens hydrolot

faut donc rejeter formellement "estavello" ho ue ia intéracure géographique et hydroles

Cependant, son succès prouve d'abord que l'on a besoin d'un terme simple pour désigner une cavité karstique à fonctionnement hydrique alternatif, dite parfoir "perte-émergence", ensuite qu'aucun pays ne dispose de ce terme dans sa langue nationale. La proposition que nous allons faire nécessite l'étude de la deuxième source à laquelle nous avons fait allusion en introduction. 


\section{LA SOURCE DE L'INVERSAC}

Cette source a fait l'objet d'innombrables descriptions. Il semble que sa première étude tant soit peu sérieuse soit celle d'Astruc, dans son "Mémoire pour l'Histoire Naturelle de la Province du Languedoc", datant de 1740. On pourrait encore citer les observations de Marcel de Serres et Louis Figuier, publiées à Montpellier en 1848, et qui ont l'avantage de réduire la part de l'imagination dans l'explication d'un fonctionnement hydrique à première vue surprenant.

Son nom d'Inversac provient peut-être de l'Occitan "Enversar", qui signifie renverser, mais la plupart des auteurs le font carrément remonter au Latin "Inversae aquae", les eaux qui s'inversent, ce qui est en somme une bonne définition du phénomène anciennement observé dans la source, comme nous allons voir ci-après.

Elle se localise $1500 \mathrm{~m}$ à l'Est de Balaruc-les-Bains, dans le quartier dit aujourd'hui Balaruc-les-Usines, $3 \mathrm{~km}$ au Nord de la ville de Sète, en bordure de l'étang (ou bassin) de Thau, qui est au niveau de la Méditerranée et en relation directe avec elle. Son exutoire est dans les calcaires miocènes, au contact de l'extrémité des calcaires jurassiques qui forment le chainon de la Gardiole entre Montpellier et Sète. Le bassin émissif débouchait autrefois sur le minuscule ruisseau de Colobres, qui n'avait guère qu'une vingtaine de mètres de longueur entre rocher et rivage de l'étang, le tout à la cote zéro, non troublée ici par des marées,

Actuellement, un chenal maritime et de larges quais édifiés devant une usine qui a capté l'eau de la source, l'ont définitivement séparée de l'étang, mais le fonctionnement hydrique antérieur avait été sérieusement

SW

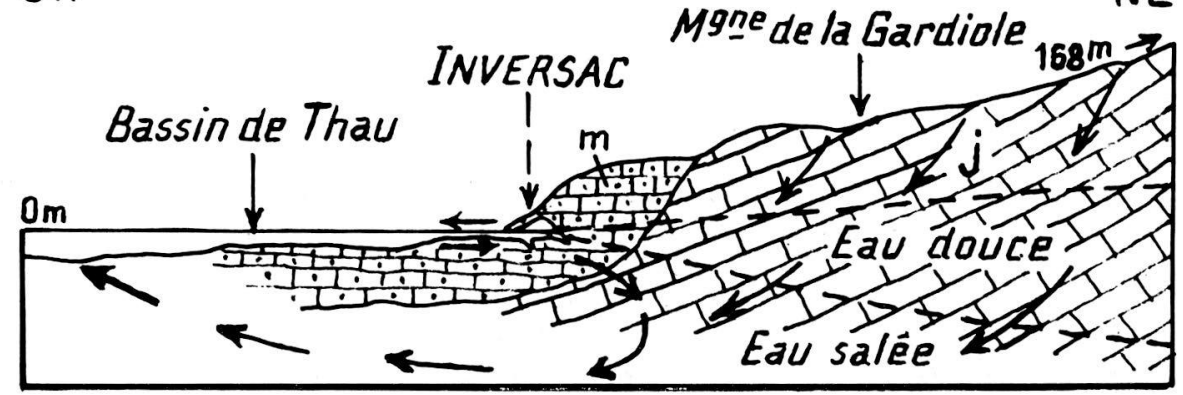

Fig. 3 - Schéma du fonctionnement hydrologique de l'Inversac.

$\mathrm{j}=$ calcaires et dolomies jurassiques; $\mathrm{m}=$ calcaires molassiques miocènes. 
étudié. Les anciens auteurs disaient qu'après un écoulement normal vers l'extérieur pendant l'hiver, au commencement de l'été, les eaux de l'étang se précipitaient sous terre avec une telle impétuosité que l'on entendait à une distance assez éloignée le bruit de leur engouffrement! Même en faisant la part de l'exagération, on doit reconnaître la réalité du phénomène puisqu'en 1925, à la suite de trois années de sécheresse persistante, l'étang se déversait dans l'Inversac à raison d'environ 10.000 mètres cubes par jour. L'eau de l'étang titrait alors 36 grammes de chlorure de sodium par litre; l'eau de la source ne renfermait pourtant que 10 grammes en septembre et atteignait en octobre le maximum de 23 grammes seulement. De là vint l'idée que, même lorsque toutes les sources du pays étaient asséchées, ou coulaient si peu que la consommation devait être sévèrement réglementée, il arrivait encore à l'Inversac une quantité d'eau douce suffisamment importante pour en tirer un utile parti.

Le problème était d'éliminer l'eau salée absolument impropre aux emplois industriels. On se mit à rechercher en amont de la source une cavité dont on supçonnait l'existence d'après un vieux plan daté du 9 octobre 1894. On découvrit en effet, à $4 \mathrm{~m}, 50$ au-dessous du sol, une petite salle, haute de $1 \mathrm{~m}, 50$, large au plus de 6 mètres et entièrement occupée par l'eau calme, profonde de 5 mètres par endroits (descendant donc plus bas que le niveau de la mer). En outre, les travaux produisirent un providentiel effondrement de rochers qui, croit-on, obstruèrent partiellement les conduits par lesquels se faisait l'absorption des eaux salées vers la profondeur. De fait, à partir de ce jour, la teneur en sel marin de la source ne cessa de décroître jusqu'à $3 \mathrm{~g}, 8$ au maximum, ce qui ne gênait plus dans la pratique.

Les quelques variations que l'on a observées par la suite continuaient à être curieusement causées par la pluie et le vent. En effet, la moindre pluie provoque une augmentation de débit qui, brassant sans doute les eaux salées préalablement engouffrées, entraîne une remontée du sel, contrairement à ce que l'on aurait pu imaginer. De même, suivant que le "mistral" (vent du NW) ou le "marin" (vent du SE) soufflent sur l'étang, celui-ci subit une sorte de crue ou au contraire de dessèchement au voisinage de la source, qui reçoit alors des infiltrations salées plus ou moins abondantes.

En juillet 1937, les conditions naturelles n'étaient encore qu'assez peu modifiées lorsque j'ai pu observer l'étrange phénomène de l'inversion du courant qui a donné son nom à l'émergence. Le flot d'eau salée pénétrant dans le ruisseau de Colobres refoulait d'abord l'eau douce qui conti- 
nuait à sortir ển s'élevant progressivement. Ensuite, l'eau de source gagnait l'extérieur en coulant en surface, tandis que l'eau salée courbait les algues vertes de la profondeur et se précipitait en sens inverse vers les fissures du rocher dans lesquelles elle s'engloutissait.

Bien entendu, comme mes prédécesseurs, j'ai imaginé d'ingénieux mécanismes pour expliquer ce phénomène, notamment une liaison possible avec les sources thermales de Balaruc-les-Bains (température atteignant $47^{\circ}$ ), ainsi qu'avec la source de l'Abysse (ou de la Vise) qui jaillit violemment au fond de l'étang de Thau à $3 \mathrm{~km}$ de l'Inversac. Mais depuis, les études sérieuses faites en Provence à la source sous-marine de Port-Miou, puis à la Roubine de Vic-la-Gardiole non loin de l'Inversac, tout aussi bien que celles consacrées aux célèbres "moulins de la mer" d'Argostoli et à plusieurs autres pertes-émergences du littoral hellénique, ont montré qu'il s'agissait d'un fonctionnement hydrique assez normal, qui s'explique simplement par la différence de densité entre l'eau douce et l'eau de mer, tandis que leur remontée commune peut se produire n'importe où vers le large.

\section{CONCLUSION GÉNÉRALE}

Bien que le cas qui vient d'être considéré soit un peu particulier et que le fonctionnement ait cessé d'être observable, le nom de la source paraît tellement favorable qu'il y a probablement intérêt à en généraliser l'emploi. Je reprends donc les termes d'une note publiée en 1971 dans Spelunca; elle servira de conclusion à cette petite révision historique des "mésaventures" de deux sources célèbres:

Un INVERSAC est une cavité karstique alternativement absorbante ou émissive, selon les saison. L'inversac peut être horizontal ou vertical, pénétrable ou impénétrable et fonctionner d'une façon pérenne ou temporaire. L'inversion des eaux, qui explique le nom de son prototype languedocien, peut avoir des causes variées qui n'interviennent pas dans la définition. Le terme d'inversac doit remplacer celui d'estavelle qui était employé a tort. 


\section{BIBLIOGRAPHIE}

Astruc, 1740 - Mémoires pour l'Histoire Naturelle de la Province du Languedoc, Paris, Guillaume Cavelier.

Cvijıc J., 1960 - La géographie des terrains calcaires, Ac. Serbe des SC. et des Arts, Monographies, t. 341, Classe des Sc. mathématiques et naturelles, n. 25, Beograd (oeuvre posthume, en français).

FOURNET J., 1859 - Hydrographie souterraine, Mém. Acad. impériale Sciences, BellesLettres et Arts, Lyon, Classe des Sciences, t. 8, $2^{\circ}$ série, p. 211-296.

GÈzE B., 1938 - Les sources mystérieuses des monts de la Gardiole (Hérault), La Géographie, 69, n. 4, p. 193-208.

GÈzE B., 1971 - Problèmes de terminologie spéléologique, Spelunca, $4^{\circ}$ serie, $11^{\circ}$ Année, n. 4 , p. $28-30$.

MARgat J., 1967 - Terminologie hydrogéologique, Chronique d'Hydrogéologie, B.R.G.M., n. 10, p. 126 (pour le mot "estavelle").

MARTel E.A., 1894 - Les Abimes, Paris, Delagrave, 580 p.

SERRES MARCEL DE, ET FIGUIER LOUIS, 1848 - Nouvelles observations sur la source thermale de Balaruc, Montpellier, Ricard Frères, 24 p.

SWEETING MARJORIE, 1972 - Karst Landforms, London, Macmillan, 362 p. 